\title{
The intercalated reinforcement technique: Learning without differential total reinforcement between groups*
}

\section{PHILIP J. BUSHNELL, KENNETH R. HENRY† and ROBERT E. BOWMAN

\author{
Regional Primate Research Center \\ University of Wisconsin, Madison. Wisconsin 53706
}

An intercalated reinforcement technique is described. The method equates number of trials, response topography, sensory exposure, and frequency of reinforcement between reversal $S$ s overtrained on a discrimination and then trained on reversals and nonreversal Ss similarly overtrained but continued on the same discrimination. In extending previous successful work in the rat, monkeys were trained on spatial discrimination and reversal tasks in the WGTA at $50 \%, 75 \%$, and $100 \%$ reinforcement in order to test the efficacy of learning by this procedure, and to compare the method with learning obtained by a more classical procedure. Learning rates were the same by both procedures. This technique, therefore, appears useful for investigating neurochemical (or other) correlates of learning unconfounded by performance or sensory effects.

To study neurochemical correlates of learning, most workers have compared neurochemical measurements between groups of learning and nonlearning animals. This paradigm clearly requires that the two groups differ only in the characteristic of learning, and that the groups be equated (or control conditions provided) for all other variables, including those affecting motor output, sensory input. reinforcement rates, etc. This requirement is quite difficult to realize and has perhaps best been approximated in a neurochemical comparison of animals learning discrimination reversals vs animals performing the equivalent, previously-learned, discrimination (Bowman \& Strobel, 1969; Kottler, Bowman, \& Haasch, 1972).

Ihese workers overtrained rats in an automated Y-maze on a single spatial discrimination for water at a 75\% reinforcement ratio. After a rat made a choice response to either one of the two goalboxes, it had to return to the startbox to reset the mechanism for delivering reinforcers in the correct goalbox. Reinforcers were delivered in the startbox on the $25 \%$ of the trials that they were not available in the correct goalbox. This training established the original learning, and also adapted all Ss to the apparatus, thereby eliminating

\footnotetext{
*Publication Number 13-001 of the Wisconsin Regional Primate Research Center. This research was supported by National Institutes of Health Grant RR-00167 to the Wisconsin Regional Primate Research Center. We also wish to thank Miss Kathleen Thompson for her assistance in animal testing. + Now at the University of California at Davis.
}

any subsequent novelty effects. A 60-min experimental session was then run following which the rats were sacrificed and neurochemical measurements were taken. During the experimental hour, the control rats were reinforced for the original discrimination and continued to perform with negligible errors. The reversal rats, however, were reinforced at $75 \%$ for repeatedly reversing their spatial discrimination to a criterion of nine correct responses in ten trials (Ss averaged two to five reversals during the experimental hour in different experiments). Thus, both groups performed highly similar responses and had highly similar exposure to the maze stimuli. To equate the groups more completely, an additional problem had to be solved. Reversal Ss, by making errors, would have received far fewer water reinforcements than the control rats. The lesser sensory and nutritional input from the reduced water consumption of the reversal Ss would then have been confounded with their reversal learning.

One solution to the problem was to use a correction procedure to balance water intake. But this would have resulted in the reversal Ss making extra motor responses and being frequently exposed to both goal discriminanda on each trial, unlike the control Ss. Another solution was to reduce the reinforcement ratio of the control Ss to the same level achieved by the reversal animals; however, this would likely have altered the performance of the control Ss, perhaps introducing expectancy learning or partial extinction. A third technique of allowing Ss to work in the presence of free reinforcers (Neuringer, 1969; Carder \& Berkowitz, 1970) would probably have disturbed performance without precisely controlling nutrient intake.

The solution devised by Bowman \& Strobel (1969) was to deliver a reinforcer in the startbox on any trial of the experimental session on which the $S$ s were not reinforced at the goalbox: startbox reinforcement followed goalbox errors and also followed that $25 \%$ of the trials when the correct goalbox was not reinforced. This meant that both the reversal Ss and the control Ss received exactly one reinforcement for each circuit of the maze, regardless of the choice responses made. It also meant that similar durations of delay of reinforcement occurred for both groups, thereby equating the durations during which frustration from delay of reinforcement might operate. The only delay difference between the groups was that the reversal rats experienced more reinforcement delays following choice responses (due to errors) whereas control rats experienced equivalently more reinforcement delays 
following startbox responses. With this innovation, the reversal task essentially equated both groups for most nonlearning (motor and sensory) characteristics.

The efficacy of the above maze task to equate for so many nonlearning factors led to the development of an analogous task for the monkey based on a spatial discrimination in the WGTA. The WGTA analogue to the goalbox response component of the Y-maze task (defined as a choice response) consisted of a choice trial on which two identical objects were presented covering food wells on the left and right sides of a form board. The WGTA analogue to the startbox response (defined as a nonchoice response) of the $Y$-maze task consisted of a trial on which a single object (identical to the choice trial objects) was presented covering a food well in the center of the form board. On the choice trials, a correct response resulted in food reinforcement at some chosen ratio, and an incorrect response resulted in no reinforcement (the classical noncorrection reward contingencies), A single object trial followed each choice trial, and the monkey's response on that trial was reinforced if and only if no reinforcement had been received on the preceding choice trial. In a sense, each choice response was reinforced: if correct, reinforcement was immediate, if incorrect, reinforcement was delayed until the animal had responded on a succeeding nonchoice trial. A choice trial followed by a single object trial defined a trial cycle.

To use this task in a neurochemical study, for example, all monkeys would be overtrained on an original spatial discrimination, using trial cycles as defined above and reinforcing correct responses on choice trials $75 \%$ of the time. During subsequent experimental sessions, the reversal monkeys would receive trial cycles on which they would be subjected to spatial reversals on the choice trials, receiving $75 \%$ reinforcement for correct reversal responses on the choice trial and receiving reinforcement on the succeeding single object trial of the trial cycle any time reintorcement was omitted or was missed (because of an incorrect response) on the preceding choice trial. Sessions with control animals would consist of trial cycles identical to those presented to the reversal monkeys except that the originally learned spatial discrimination would continue to be reinforced on the choice trials. Thus, both groups of monkeys would be presented with the same sequence of trial cycles, resulting in highly similar responses and stimulus exposure. Both groups would receive exactly one reinforcement for each trial cycle. Any neurochemical differences observed between the groups would be correlated with reactions to the altered pattern of response-reinforcement contingencies (i.e., learning the reversals-which may involve several underlying components including extinction of the original discrimination) and would not be confounded by differences in nature, frequency, or amount of either motor responding, stimulus input, or obtained reinforcement.
A test of the intercalated reinforcement (IR) technique is described here. The test had two purposes: (1) to demonstrate that the method elicits reversal learning in the monkey and (2) to compare the rate and amount of such learning with learning under the usual noncorrection WGTA procedure, omitting intercalated trials.

\section{METHOD \\ Subjects}

Twenty feral female rhesus monkeys were used. For purposes unrelated to the present technique, animals were placed in restraining chairs for the duration of the experiment. Each animal was kept in a chair for not less than a week, and generally not more than a month. About one-half of the $S$ s had had previous experience with WGTA testing procedures. These animals were distributed equally across all conditions.

\section{Apparatus}

A modified WGTA (Harlow, 1949) was built to conform to the dimensions of the monkey chair. The stimulus tray, $59 \mathrm{~cm} \times 22 \mathrm{~cm}$, contained three foodwells $15 \mathrm{~cm}$ apart, center to center, at the S's end of three shallow runways $7 \mathrm{~cm}$ wide and $20 \mathrm{~cm}$ long (positioned left, center, and right on the form board). Two identical gray blocks, $3.5 \mathrm{~cm}$ wide $x 5 \mathrm{~cm}$ high $x$ $6.5 \mathrm{~cm}$ deep, fixed on flat plastic plates that slid in the runways, served as covers for the food wells. $S$ was required to push the blocks along the runways to uncover the wells. On any trial, one block in the center position or two blocks (one in each side position) was presented to $S$

\section{Design}

Five treatment groups of four Ss each were established. These were: intercalated reinforcement trials at $75 \%$ and $50 \%$ reinforcement ratios (75I and 50I) and standard reinforcement trials at $100 \%, 75 \%$, and $50 \%$ reinforcment ratios $(100,75$, and 50 ). The $50 \%$ and $75 \%$ reinforcement schedules were determined by randomizing the order of reinforced and nonreinforced trials in blocks of ten: seven or eight trials out of ten were reinforced for the $75 \%$ conditions, and five trials out of ten were reinforced for the $50 \%$ conditions. Each test session was run a uniform 50 trials. Therefore, in the intercalated reinforcement conditions, $S$ recejved exactly one reinforcement per trial cycle and 50 reinforcements per session, irrespective of its performance or the reinforcement ratio designated to its treatment group. The standard groups, by contrast, were given no single object trials. They received a sequence of 50 choice trials of two identical blocks in the side positions, with the total amount of received reinforcement dependent upon the number of correct responses made by each $S$ and the reinforcement ratio of the treatment group.

\section{Procedure}

Each $S$ was placed in a restraining chair while briefly anesthetized with Surital (thiamylal sodium, P.D.). The monkey was then given 2-3 days to adapt to the chair and to learn to work the WGTA from its restrained position. $S$ was next given preliminary training on the WGTA until it learned to push the blocks from the food wells for reinforcment. At this point. original learning was begun; the $S$ was trained to either the right or left position to a criterion of $90 \%$ or more correct for two consecutive 50-trial sessions. This generally required three to four sessions. Subsequently, $S$ began reversal learning, i.e., the opposite position was reinforced until a criterion of nine correct trials in a ten-trial block was reached. Then the position of reinforcement was again reversed and nun until $S$ achieved the nine-out-of-ten criterion for seven successive reversals. 


\section{RESULTS}

Figure 1 shows the mean number of trials to criterion plotted for each reinforcement condition for the original learning (designated Reversal 0 ) and for the seven reversals. Original learning appears unaffected by differences in reinforcement ratio or by the use of intercalated reinforcement, and analysis of variance with orthogonal contrasts of the conditions during the original learning indicated no significant effects (all Fs $<1$ ).

Figure 1 also suggests that the ratio affected the animals' patterns of acquisition of reversal learning set. An analysis of variance with orthogonal contrasts of the reversal learning data indicated that, across Reversals 1.7. the 100 group was significantly superior to the other conditions combined (i.e. the 75,75I, 50, and 501 conditions; $F=6.25$, df $=1 / 15, p<.05)$. In addition, the 50 and 501 conditions combined had a significantly steeper linear trend $(\mathrm{F}=7.64: \mathrm{df}=1 / 15, \mathrm{p}<.05)$ and more bowed quadratic trend $(F=7.24, \mathrm{df}=1 / 15$, $\mathrm{p}<.05)$ than the 75 and $75 \mathrm{I}$ conditions combined. The intercalated reinforcement procedure exerted no significant effect upon the monkey's patterns of acquisition of reversal learning set, since the ANOVA indicated no significant differences in either main effects or trends accross reversals when comparing the 75 with the $75 \mathrm{I}$ condition, or the 50 with the $50 \mathrm{I}$ conditions.

\section{DISCUSSION}

As described, the IR technique precisely equates such performance variables as the kind and number of trials, of responses, of sensory exposures. and of reinforcements between groups performing at different rates of correct responding. This is not true of any alternative procedures available for studying types of discrimination learning (see lntroduction). Although the present IR technique accomplished this within the context of reversal learning set. it appears readily possible to extend the procedure to discrimination based on object quality as well. Furthermore. Shusterman (1962) has shown that reversal learning set in the chimpanzee yields almost perfect transfer to obecf quality learning set, suggesting that reversal learning set is not markedly different in this case from other types of discrimination leasning. Also. the overall effect of reinforcement ratio on reversal learning was grossly similar to that described earlier for acquisition of object quality leaming set (Bowman. 1963).

The question of how the intercalated reinforcement technique works, that is. why it produced no decrement in performance on the choice trials. Was not investigated. since we were mainly interested in it as a technique. It may possibly be an example of Harlow's (1949) concept of "functional isolation" of leaming set. derived from the fact that monkeys can solve two types of learnig set problems simultaneously:.

The intercalated reinforcement technique can be viewed as two "problems" administered together-the choice trials representing one "problem" and the nonchoice trials representing another. There is little evidence in the data that the "choice problem" interacts with the "nonchoice problem" since the means of the 75 and 751 groups and those of the 50 and 501 groups follow quite similar contours across reversals. A possible explanation for the minimal effect of intercalated trials on

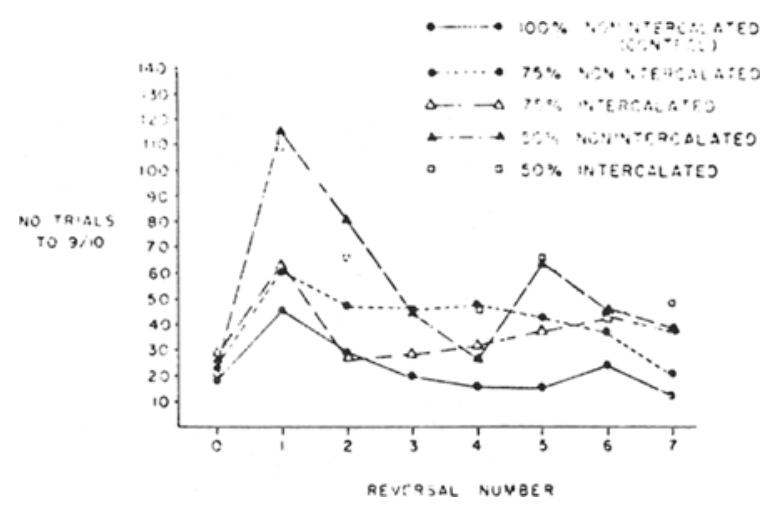

Fig. 1. Mean trials to criterion for the original learning (Reversai 0 ) and for the seven reversals for all conditions of the study.

choice trial performance is that the single object reinforcement was delayed long enough following an incorrect choice trial that it was not sufficient to reinforce the learning of incorrect responses on the choice trials.

In assessing neurochemical correlates of learning. either learning on the choice trials or learning on the intercalated trials, or both, could produce neurochemical effects. This is not serious at an early stage of the neurochemical study of learning, since either or both effects would represent learning parameters. At a later stage of analysis, this possible learning confound might be disentangled by studying single object trials alone, in which reinforcement patterns were the same as observed during intercalated learning. Another possibility would be to compare neurochemistry under the IR procedure with that under alternate procedures such as noncorrection, forced trials etc. However. these latter techniques potentially include confounds of their own. which appear to us more difficult to analyze than does the IR procedure. Finally. reversal learning itself may include components (such as extinction processes) which differentiate it from other learning tasks both behaviorally and neurochemically, and these issues remain to be explored.

In summary, the IR procedure appears to permit a study of learning parameters unconfounded by any performance differences. This does not appear possible with any other currently available procedure. Although we have conceptualized the usefulness of the IR technique within the context of neurochemical studies of learning, there may well be other applications which will occur to the reader.

\section{REFERENCES}

Bowman, R. E. Discrimination learning set performance under intermittent and secondary reinforcement. Journal of Comparative \& Physiological Psychology, 1963, 56, 429-434.

Bowman, R. F., \& Strobel, D. A. Brain RNA metabolism in the rat during learning. Journal of Comparative \& Physiological Psychology, 1969,67, 448-456.

Carder, B., \& Berkowitz, K. Rats' preference for earned in comparison with free food. Science, 1970. 167, 1273-1274.

Harlow, H. F. The formation of learning sets. Psychological Review, $1949,56.51 .65$

Kottler, P. D., Bowman, R. E., \& Haasch, W. D. RNA metabolism in the rat brain during learning following intravenous and intraventricular injections of H-cytidine. Physiology \& Behavior, 1972, 8, 291-297.

Neuringer, A. J. Animals respond for food in the presence of free food Science, $1969,166,399-401$.

schusterman. R. J. Transfer effects of successive discrimination-reversal training in chimpanzees. Science. 1962. $137,422-423$

(Received for publication January 15, 1973: revision received A pril 26,1973 .) 